\title{
PRIMARY HYPERALDOSTERONISM: CLINICAL AND THERAPEUTIC APPROACH OF A CENTER
}

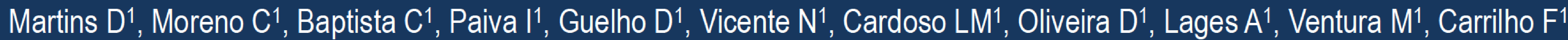

'Endocrinology, Diabetes and Metabolism Department of Coimbra Hospital and University Centre, Portugal

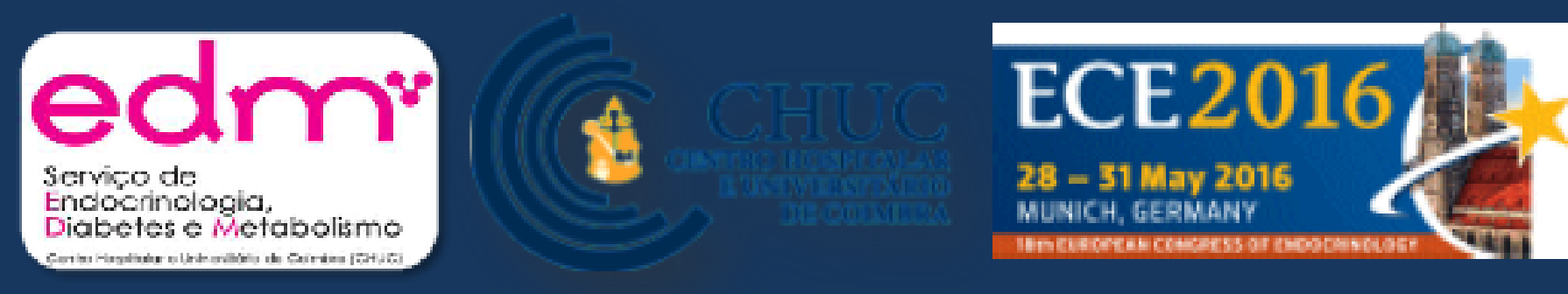

Introduction: Primary hyperaldosteronism (PHA) is the main cause of secondary hypertension, with a prevalence estimated between 6-20\% in patients with resistant hypertension. Clinical suspicion is critical, especially if aldosterone-to-renin ratio (ARR) $>25$, however the diagnosis is dependent on confirmatory evidence, including aldosterone suppression tests.

Methods: A retrospective study including 44 patients with suspected PHA, identified between 2010 and 2015 at the Endocrinology, Diabetes and Metabolism Department of Coimbra Hospital and University Centre, Portugal, was performed. Diagnostic criteria and treatment response were evaluated.

\section{Results}

\section{$\mathrm{N}=44$ patients}

Suspicion of secondary hypertension

Refractory hypertension

Hypertension with hypokalemia

Hypertension and adrenal mass

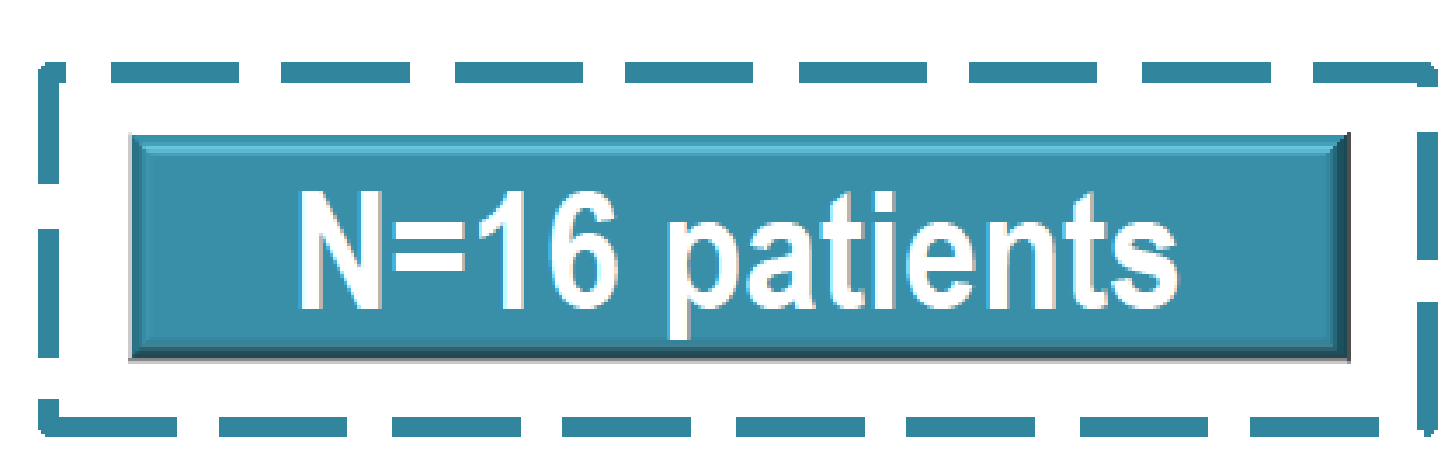

Men: $n=9$ (56.25\%); Women: $n=7(43.75 \%)$ Median age $=60.5$ years

\section{1) Clinical presentation}

\section{Clinical presentation}

Refractory hypertension

Hypertension and hypokalemia

Hypertension and adrenal mass

$\begin{array}{cc}\text { n } & \text { \% } \\ \mathbf{9} & \mathbf{5 6 . 2 5} \\ 6 & 37.5 \\ 1 & 6.25\end{array}$

\section{8 patients excluded}

- 15 patients: ARR < 25

- 11 patients: negative infusion saline test (IST)

- 2 patients: negative captopril test

\section{2) Hypertension (HT) - characterization}

Median duration: 9.00 years

$\begin{array}{cccc}\text { Stage of HT } & \text { I } & \mathbf{1 4} & \mathbf{8 7 . 5} \\ & \text { II } & 1 & 6.3 \\ & \text { III } & 1 & 6.3 \\ & \text { IV } & 0 & 0\end{array}$

$$
\text { A }
$$

$\begin{array}{clcc}\text { Associated Diseases } & \text { N } & \% \\ \text { Cardiovascular } & \text { Stroke } & 0 & 0 \\ & \text { EAM } & 1 & \mathbf{6 . 2 5} \\ \text { AF } & 0 & 0 \\ \text { CHF } & 0 & 0\end{array}$

Median number of antihypertensive drugs $\mathrm{N}=1.75(\min 1 ; \operatorname{máx} 3)$

Chronic renal disease

Hypertensive retinopathy

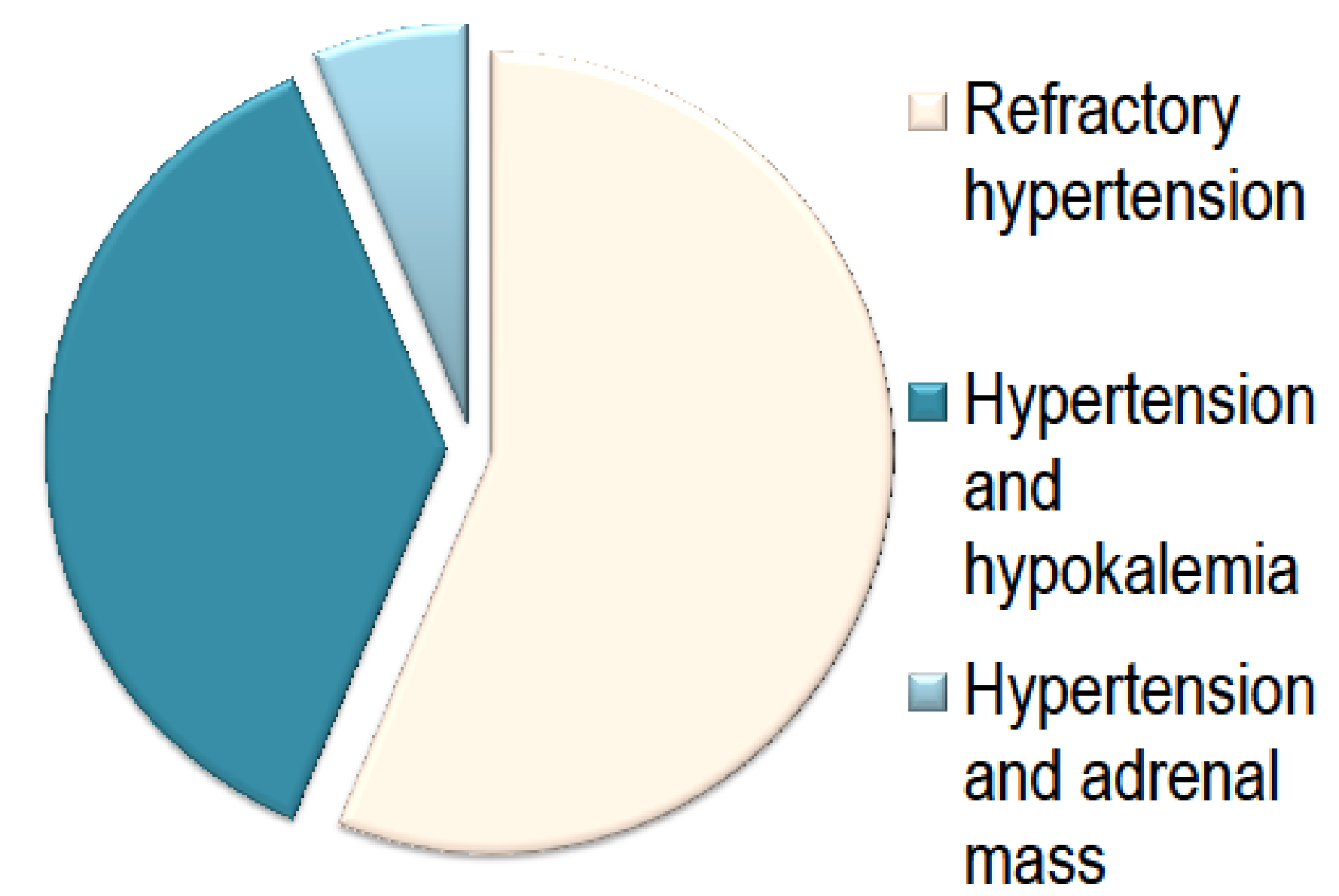

\section{3) Analytical evaluation}

\begin{tabular}{|c|c|c|c|}
\hline Parameter & Result* & VR & $\begin{array}{l}14 \text { patients with } A R R>25 \\
\text { and positive aldosterone }\end{array}$ \\
\hline Plasmatic renin $\mu \mathrm{Ul} / \mathrm{mL}$ & 1.65 & $7-76$ & supression test \\
\hline Plasmatic aldosterone $\mathrm{pg} / \mathrm{mL}$ & 213.00 & $40-310$ & \\
\hline ARR & 131.44 & - & 2 patients with $A R R<25$ \\
\hline Potassium mmol/L & 3.45 & $\begin{array}{c}3.50-5.10 \\
{ }^{*} \text { Median }\end{array}$ & $\begin{array}{c}\text { and positive aldosterone } \\
\text { supression test }\end{array}$ \\
\hline
\end{tabular}

ACE inhibitors $\quad 1(6.3 \%)$

Calcium channel blockers $\quad 11(68.8 \%)$

Beta-blockers

$3(18.8 \%)$

$4(25 \%)$

Spironolactone $\quad 1(6.3 \%)$

Antiadrenergics $\quad 2(12.5 \%)$

3) Radiological evaluation

\begin{tabular}{|c|c|c|c|c|}
\hline Localization & & $n$ & $\%$ & - \\
\hline Unilateral & Right & 6 & 37.5 & - Unilateral-Left \\
\hline & Left & 6 & 37.5 & \\
\hline Bilateral & & 2 & 12.5 & Bllateral \\
\hline Without adrenal I & sion & 2 & 12.5 & \\
\hline Averag & size: 1 & & & $\begin{array}{l}\text { Without adrenal } \\
\text { mass }\end{array}$ \\
\hline
\end{tabular}

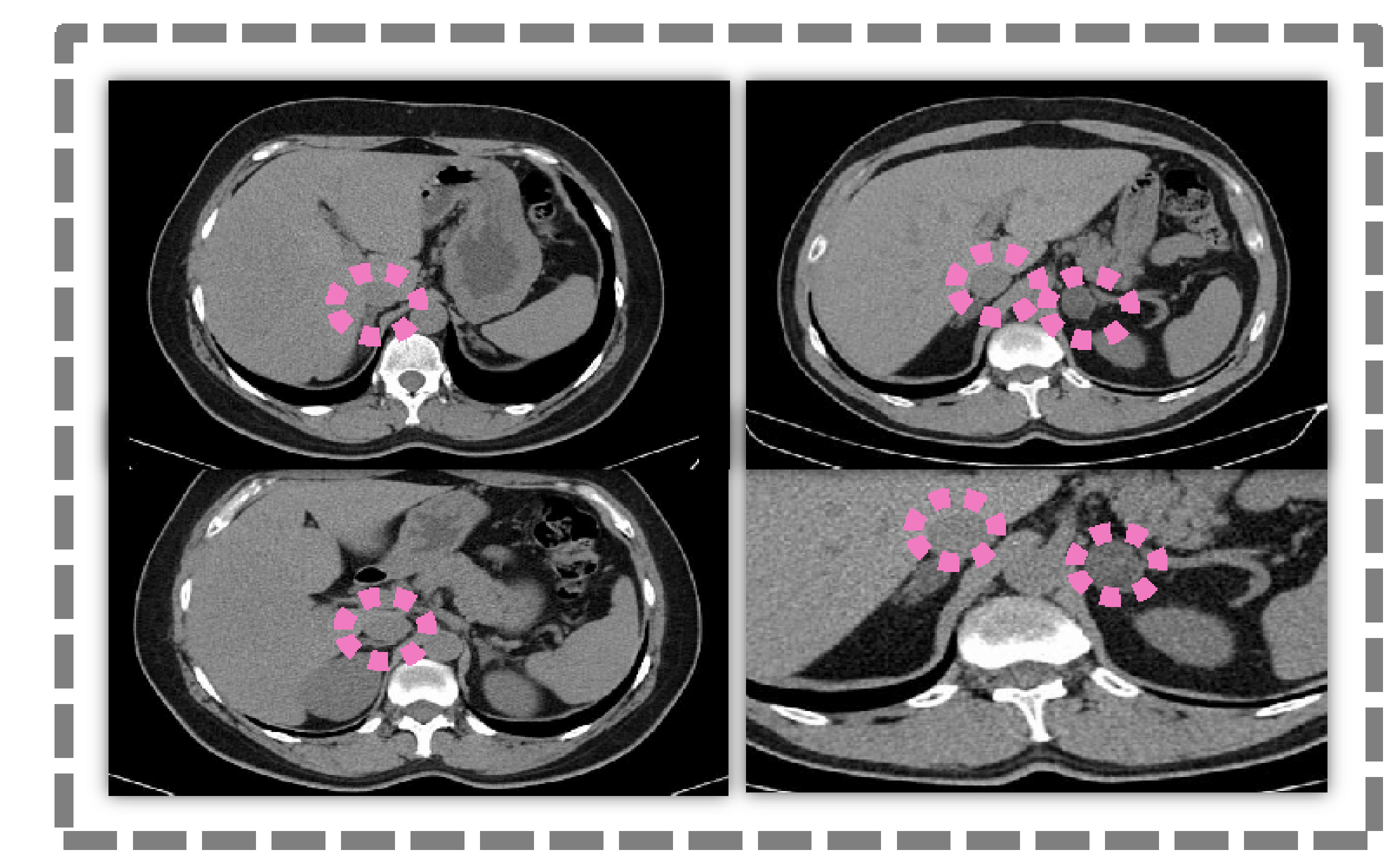

4) Treatment

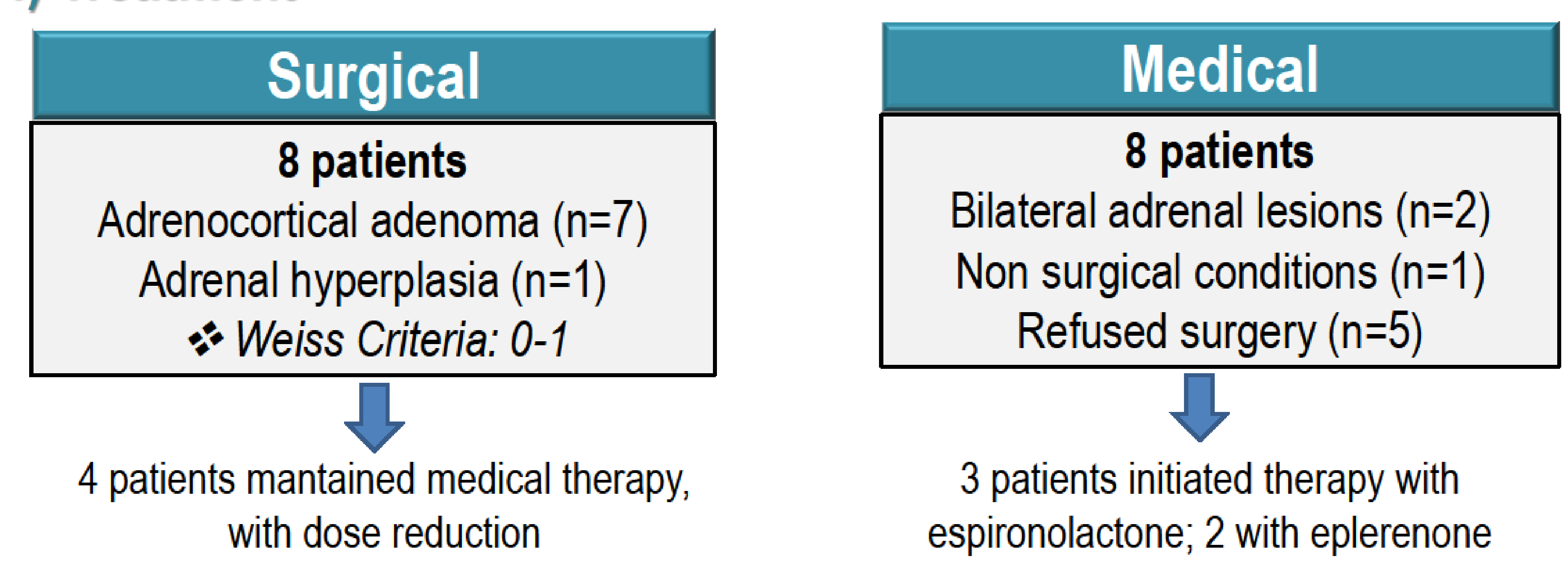

5) Follow-up (6 months)

Conclusions: On follow-up at 6 months, it was found a significant overall reduction in the levels of systolic BP and the number of antihypertensive drugs. Surgical treatment produced a more significant reduction in the levels of systolic BP compared with medical treatment alone and the reduction of serum aldosterone levels was also higher in the group undergoing surgery. Further studies are needed in order to clarify the ideal cut-off of ARR, as well as the benefits of medical vs surgical treatment.

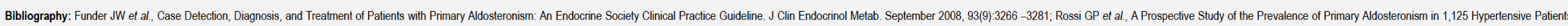

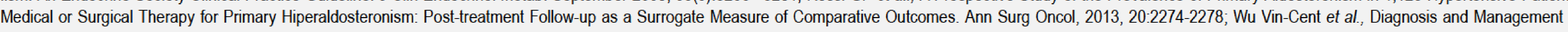
Primary Aldosteronism. Acta Nephrologica, 2012, 26(3): 111-120; Muth A et all, Systematic review of surgery and outcomes in patients with primary aldosteronism. BJS 2015; 102: 307-317; Jansen PM et al., Test 